Int. J. Morphol.,

32(3): 1074-1078, 2014

\title{
Diferencias Osteológicas entre los Subgéneros Profundulus y Tlaloc (Teleostei: Profundulidae)
}

\author{
Osteological Differences between the Subgenus Profundulus and Tlaloc (Teleostei: Profundulidae)
}

\author{
Alfonso Ángel González-Díaz*; Edmundo Díaz-Pardo*; Miriam Soria-Barreto* \& Emilio Martínez-Ramírez ${ }^{* * * *}$
}

GONZÁLEZ-DÍAZ, A. A; DÍAZ-PARDO, E.; SORIA-BARRETO, M. \& MARTÍNEZ-RAMÍREZ, E. Diferencias osteológicas entre los subgéneros Profundulus y Tlaloc (Teleostei: Profundulidae). Int. J. Morphol., 32(3):1074-1078, 2014.

RESUMEN: El género Profundulus es uno de los grupos de peces más antiguos, distribuidos al norte de América Central en México, Guatemala y Honduras. No obstante, son pocos los trabajos que se han realizado para conocer el número de especies que lo conforman y sus relaciones filogenéticas. Históricamente se reconoció que este taxón estaba conformado por dos grupos naturales, actualmente considerados como los subgéneros Profundulus y Tlaloc. Recientes investigaciones genéticas han revelado un alto grado de diferenciación entre ambos taxones y algunas de sus especies, lo cual probablemente este asociado a cambios morfológicos. De esta manera, se realizó la comparación osteológica entre seis especies de los subgéneros Profundulus y Tlaloc (Cyprinodontidae: Profundulidae). Se revisaron 17 huesos ubicados en el cráneo y la cintura pélvica, se encontraron diferencias cualitativas en el mesetmoides, vómer, lacrimal, premaxilar, articular, cuadrado, interopérculo y basipterigio. Los patrones de variación observados permiten diagnosticar ambos subgéneros, corroborando lo encontrado en estudios morfológicos y moleculares. Las evidencias permiten suponer que ambos taxones podrían ser reubicados como géneros, como previamente se había establecido.

PALABRAS CLAVE: Cyprinodontiformes; Centroamérica; Peces dulceacuícolas; Osteología comparada; Neurocraneo; Branquiocraneo.

\section{INTRODUCCIÓN}

El género Profundulus (Hubbs, 1924) es un grupo de peces dulceacuícolas originarios y endémicos de Centroamérica que habitan en el sur de México. Pertenecen a la familia Profundulidae que es la más primitiva del orden Cyprinodontiformes. Se estima que divergió durante las épocas del Plioceno o Mioceno de la era Cenozoica, además presentan un número grande de caracteres plesiomorficos parecidos (Hubbs; Miller, 1955; Parenti, 1981; Doadrio et al., 1999). Su distribución comprende las vertientes del Atlántico y Pacífico al norte de la parte central de América en México, Guatemala y Honduras; en el Atlántico desde la cuenca del río Papaloapan (México) hasta las cuencas de los ríos Motagua y Polochic (Guatemala); en el Pacífico de la cuenca río Balsas (México) a la cuenca río Lempa (Honduras) (Hubbs; Miller; Parenti; Page \& Burr, 1991; Espinosa et al., 1993; Doadrio et al.; Martínez, 2000; Nelson, 2006). Está integrado por nueve especies, distribuidas en los subgéneros Profundulus (P. balsanus, $P$. guatemalensis, $P$. kreiseri, P. oaxacae y $\mathrm{P}$. punctatus) y Tlaloc (P. candalarius, P. hildebrandi, $P$. labialis y $P$. portillorum) (Miller; Doadrio et al.; Miller et al., 2005; Matamoros \& Schaefer, 2010; Matamoros et al., 2012).

El establecimiento de estos dos subgéneros originalmente se sustentó en diferencias morfológicas externas; las especies del subgénero Profundulus presentan una mancha humeral de color obscuro en ambos lados del cuerpo y tienen más de la mitad de la base de la aleta caudal cubierta de escamas, mientras que los miembros de Tlaloc carecen de mancha humeral y no presentan escamas por detrás de la mitad de la aleta caudal (Álvarez \& Carranza, 1951; Miller). Análisis moleculares basados en enzimas y citocromo $b$ han confirmado que las distancias genéticas entre ambos subgéneros y algunas de sus especies son considerables y se reflejan en las relaciones filogenéticas de la familia Profundulidae (Doadrio et al.; Matamoros et al.).

* El Colegio de la Frontera Sur, Unidad San Cristóbal, Chiapas, México.

** Facultad de Ciencias Naturales, Universidad Autónoma de Querétaro, Querétaro, México.

**** Centro Interdisciplinario de Investigación para el Desarrollo Integral Regional, Unidad Oaxaca, Oaxaca, México. 
A pesar que las características moleculares y la morfología externa que permiten distinguir ambos subgéneros, aún no se ha determinado el valor taxonómico de las estructuras óseas, las cuales tienen gran importancia en las clasificaciones de peces, generalmente por arriba del nivel de especie. En este sentido, existen trabajos donde se han descrito algunas características osteológicas del género Profundulus. Uyeno \& Miller (1962) compararon algunas estructuras óseas para separar el género fósil Empetrichthys de los géneros actuales Crenichthys, Profundulus y Fundulus.

Asimismo, dentro del orden Cyprinodontiformes, al que pertenece el género Profundulus, se han realizado algunos análisis osteológicos para proponer las relaciones filogenéticas entre las familias y su clasificación (Parenti; Costa, 1998). Sin embargo, al contrastar las hipótesis filogenéticas ambos estudios se observan inconsistencias, debido principalmente a diferencias en la descripción de algunos huesos y su interpretación evolutiva (Costa).

De esta manera, el propósito del presente trabajo fue comparar algunas piezas óseas de seis especies del género Profundulus, para evaluar su utilidad en el reconocimiento de los subgéneros Profundulus y Tlaloc, así mismo, contribuir al esclarecimiento de su estatus taxonómico y clasificación.

\section{MATERIAL Y MÉTODO}

Se seleccionaron diez ejemplares sexualmente maduros (hembras y machos) de seis especies: Profundulus (T.) candalarius, $P$. (T.) hidebrandi, $P$. (T.) labialis, $P$. (P.) oaxacae y $P$. (P.) punctatus, con excepción de $P$. (P.) guatemalensis, de la cual sólo se dispuso de dos organismos.

Los números de catálogo y los ejemplares empleados son señalados entre paréntesis, fueron los siguientes: Profundulus candalarius ECOSC 3997 (6), 3998 (4); P. guatemalensis UMMZ 166681 (2); P. hildebrandi ECOSC 2820 (1), 2821 (1), 4007 (8); P. labialis ECOSC 3927 (10); P. oaxacae CIIDIR-OAX 187 (5), 188 (5); P. punctatus ECOSC 3985 (10).

Con un vernier electrónico de precisión $(0,01 \mathrm{~mm})$, se tomaron datos biométricos de cada ejemplar (longitud patrón, longitud cefálica, altura máxima y altura mínima). Los organismos fueron descamados y eviscerados, para posteriormente ser procesados siguiendo la técnica de transparentación y tinción de Hollister (1934). Con el uso de una lupa estereoscópica se realizó una revisión general del esqueleto para conocer la variación intraespecífica y seleccionar que estructuras serían descritas.
Se desarticularon 17 piezas óseas de cada organismo, 16 ubicadas en el cráneo (lacrimal, premaxilar, maxilar, dentario, articular, autopalatino, mesopterigoide, cuadrado, simpléctico, hiomandibular, preopérculo, opérculo, interopérculo, subopérculo, mesetmoides y vómer) y una de la cintura pélvica (basipterigio). Además, se revisaron características de vértebras, radios y espinas. De cada pieza se realizaron dibujos con el empleo de una cámara clara Zeiss y se analizó su variación intraespecífica para buscar atributos cualitativos discriminantes entre las especies. Para las descripciones de las estructuras pares se utilizaron las ubicadas en el lado izquierdo, conservando su posición natural, asimismo se siguió la nomenclatura y terminología empleada en los trabajos de Parenti y Costa.

\section{RESULTADOS}

La comparación osteológica entre las seis especies mostró que al interior de los subgéneros Profundulus y Tlaloc, no existen diferencias cualitativas evidentes y se presenta un patrón de variación constante. Sin embargo, al comparar entre los subgéneros si es posible reconocer características cualitativas que los definen y separan claramente. De esta manera, se logró diagnosticar que las especies que integran el subgénero Profundulus son Profundulus (P.) guatemalensis, $P$. (P.) punctatus y $P$. (P.) oaxacae, mientras que el subgénero Tlaloc lo forman $P$. (T.) candalarius, $P$. (T.) hildebrandi y $P$. (T.) labialis. A partir de estos resultados se elaboraron los esquemas usando ejemplares representativos de cada subgénero, en el caso del subgénero Profundulus se usó a $P$. (P.) punctatus y $P$. (P.) guatemalensis; para el de Tlaloc a $P$. (T.) hildebrandi y $P$. (T.) labialis.

Las diferencias se observaron en ocho piezas, de las cuales seis pertenecen al esqueleto axial (neurocráneo y branquiocráneo) y una al apendicular (aletas pélvicas). A continuación se describen las diferencias encontradas en cada pieza.

\section{Neurocráneo}

Mesetmoides: En el subgénero Profundulus este hueso es pequeño, prácticamente cabe en el cuerpo del vómer y tiene forma de media luna, en tanto que en Tlaloc es considerablemente más grande y de forma ovalada, rebasando por mucho los márgenes posteriores del vómer (Fig. 1).

Vómer: En las especies del subgénero Profundulus esta pieza es de forma triangular, mientras que en Tlaloc tiene forma de "Y" (Fig. 1).

Lacrimal: En el subgénero Profundulus el margen ventral de la pieza se prolonga posteriormente, en cambio en 
las especies de Tlaloc este hueso no presenta dicha prolongación, su forma es casi cuadrada (Fig. 2).

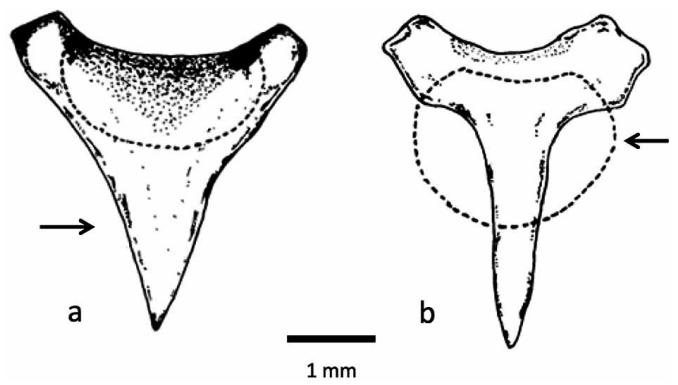

Fig. 1. Vista dorsal del mesetmoides (líneas punteadas) y vómer. a) Profundulus (P.) punctatus, b) P. (T.) labialis.
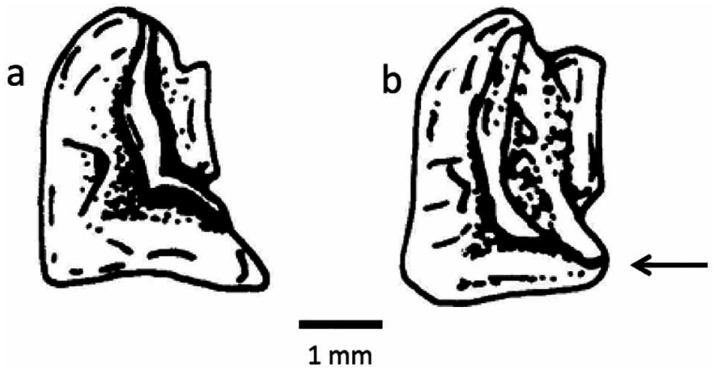

Fig. 2. Vista lateral del lacrimal. a) Profundulus (P.) punctatus, b) $P$. (T.) labialis.

\section{Branquiocráneo}

Premaxilar: En las especies de Profundulus es un hueso corto y el proceso ascendente es más ancho en su base, se adelgaza gradualmente hacia la parte posterior. Mientras que en Tlaloc la pieza es ligeramente más larga y la base del proceso es más delgada, prácticamente conserva el mismo ancho en toda su longitud (Fig. 3).

Articular: En el subgénero Profundulus el proceso ventral es notablemente más largo y ancho que en Tlaloc (Fig. 4).

Cuadrado: En el subgénero Profundulus el margen posterior del abanico es cóncavo, mientras que en las especies de Tlaloc es recto o convexo (Fig. 5).

Interopérculo: En Profundulus el margen dorsal tiene una prolongación corta dirigida hacia la parte anterior, mientras que en Tlaloc esta prolongación es más larga, rebasa el borde anterior del cuerpo de la pieza (Fig. 6).

\section{Aletas pélvicas}

Basipterigio: En las especies del subgénero Profundulus el proceso medio es considerablemente más corto que en los miembros de Tlaloc (Fig. 7).

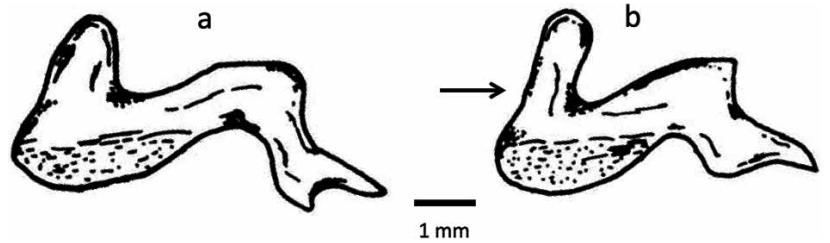

Fig. 3. Vista dorsal del premaxilar. a) Profundulus (P.) punctatus, b) $P$. (T.) labialis.
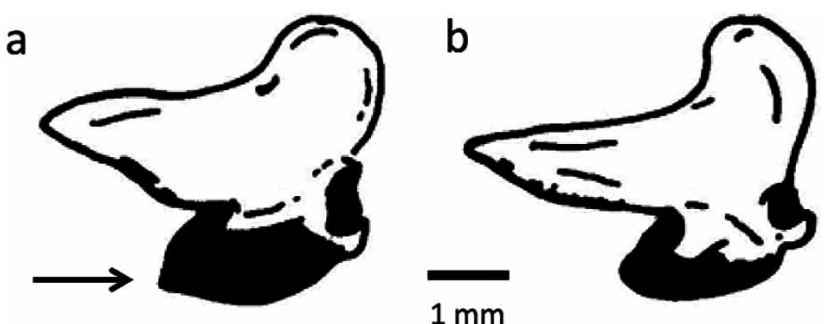

Fig. 4. Vista lateral del articular. a) Profundulus (P.) punctatus, b) $P$. (T.) hildebrandi.

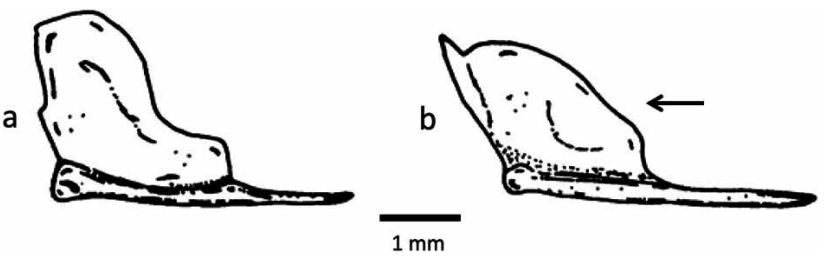

Fig. 5. Vista lateral del cuadrado. a) Profundulus (P.) guatemalensis, b) $P$. (T.) labialis.

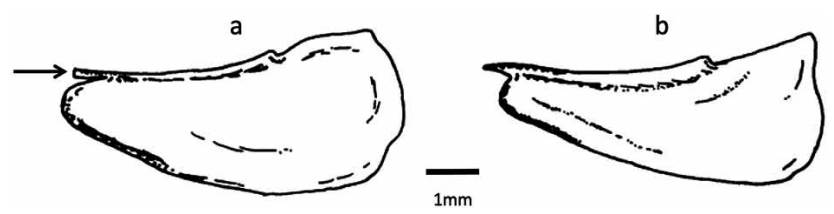

Fig. 6. Vista lateral del interopérculo. a) Profundulus (P.) punctatus, b) P. (T.) labialis.

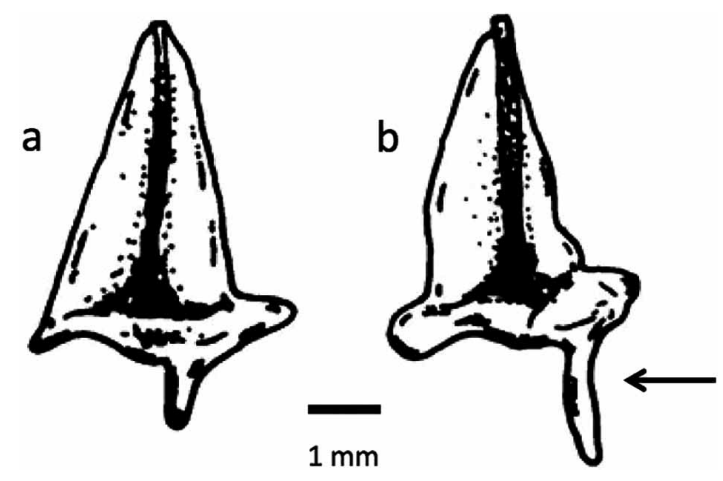

Fig. 7. Vista dorsal del basipterigio. a) Profundulus (P.) guatemalensis, b) $P$. (T.) labialis . 


\section{DISCUSIÓN}

A partir de la revisión osteológica de las seis especies del género Profundulus se encontraron dos patrones de variación, uno de ellos permite reconocer a $P$. (P.) guatemalensis, $P$. (P.) oaxacae y $P$. (P.) punctatus, mientras que el otro a agrupa a $P$. (T.) candalarius, $P$. (T.) hildebrandi y $P$. (T.) labialis. Estos patrones coinciden con los subgéneros Profundulus y Tlaloc respectivamente, robusteciendo la idea de que ambos taxones son monofiléticos (Doadrio et al.). En los casos de $P$. portillorum, $P$. kreiseri y $P$. balsanus especies que no se incluyeron en este trabajo, sus características morfológicas externas sugieren que la primera pertenece al subgénero Tlaloc y las restantes a Profundulus, sin embargo, sería conveniente confirmar esto a través de la revisión osteológica.

En contraste, se encontró que la variación osteológica cualitativa al interior de cada grupo es insuficiente para poder diagnosticar las especies. No obstante, no se debe descartar la posibilidad de que existan diferencias cuantitativas que puedan ser útiles en su reconocimiento, como se ha demostrado a través del análisis de la variación morfológica entre las especies (González-Díaz et al., 2005).

Se puede señalar que dentro del género Profundulus las características osteológicas y morfológicas permiten reconocer claramente a los miembros de los subgéneros Profundulus y Tlaloc, mientras que los atributos merísticos y morfométricos son útiles para diagnosticar a las especies (Miller; Miller et al.; González-Díaz et al.; Matamoros \& Schaefer).

Es importante mencionar que el grado de diferenciación osteológica coincide con los resultados obtenidos en los estudios moleculares para reconocer ambos subgéneros (Matamoros et al.). De esta manera, existe una probabilidad alta que las diferencias morfológicas y osteológicas que definen a los subgéneros y sus especies tengan sustento genético y puedan ser utilizadas para entender la historia evolutiva del género (Doadrio et al.).

En este sentido, las hipótesis filogenéticas propuestas para el orden Cyprinodontiformes, a partir de información osteológica muestran inconsistencias, que tienen implicaciones taxonómicas y sistemáticas para la familia Profundulidae (Parenti; Costa). Parenti consideró que las características osteológicas de la familia Profundulidae la sitúan como el representante más primitivo del suborden Cyprinodontoidei. Por el contrario, Costa encontró que las familias Goodeidae y Profundulidae son grupos hermanos y que junto con Fundulidae integran la subfamilia Funduloidea; este autor no considera que Profundulidae sea el taxón más primitivo dentro del suborden Cyprinodontoidei.

A partir de los resultados aquí expuestos, donde se deja clara la existencia de dos patrones osteológicos dentro del género Profundulus, y se propone que la explicación de las inconsistencias filogenéticas a nivel de familia, podría ser que algunas de las diferencias óseas existentes entre los subgéneros pasaron desapercibidas y fueron interpretadas como iguales para las especies de ambos taxones, de esta manera Parenti y Costa hicieron generalizaciones que finalmente condujeron a hipótesis incompatibles.

En el presente estudio se corroboran las observaciones descritas por Uyeno \& Miller, sobre la forma del mesetmoides y el vómer. El mesetmoides es pequeño con forma de media luna en los miembros del subgénero Profundulus, mientras que en Tlaloc este hueso es considerablemente mayor y circular. En tanto, el vómer es triangular en el subgénero Profundulus y en forma de «Y» en Tlaloc. Sin embargo, la descripción de Parenti considera que todos los miembros del género dicha estructura es de forma triangular. Al contrario de las observaciones de Costa, quien estableció que la forma de «Y» es distintiva de Profundulus. Así se pone de manifiesto, que ambos trabajos incluyeron la revisión de algún miembro de los subgéneros.

Una situación semejante se registró con el tamaño y forma del proceso ascendente del premaxilar, en el presente trabajo se observó que en el subgénero Profundulus esta estructura es corta y amplia en su base; mientras que en Tlaloc es alta con base estrecha. Sin embargo, Parenti generalizó para el género la forma del premaxilar encontrada en las especies del subgénero Profundulus; en tanto Costa hizo lo mismo, pero considerando la forma mostrada por las especies de Tlaloc.

La importancia taxonómica que se le ha otorgado a las características óseas y su interpretación evolutiva tienen consecuencias significativas en la construcción de hipótesis filogenéticas. Por tal motivo, sería importante incorporar está información para evaluar las relaciones de parentesco entre los miembros de la familia Profundulidae. Así como, su clasificación dentro de los Cyprinodontiformes, en particular sus vínculos con las familias Goodeidae y Fundulidae.

A pesar que la sistemática de este orden de peces es compleja, existen evidencias moleculares, morfológicas y osteológicas para afirmar que los subgéneros Profundulus y Tlaloc son linajes diagnosticables y con historias evolutivas independientes. Incluso el grado de diferenciación permite sugerir que podrían ser considerados géneros taxonómicamente válidos, como en su momento se propuso para Tlaloc (Álvarez \& Carranza). 
AGRADECIMIENTOS. Agradecemos al Sistema Nacional de Investigadores de CONACyT por el apoyo brindado. A Rocío Rodiles por la ayuda para obtener los ejemplares de Profundulus guatemalensis.

GONZÁLEZ-DÍAZ, A. A; DÍAZ-PARDO, E.; SORIABARRETO, M. \& MARTÍNEZ-RAMÍREZ, E. Osteological differences between the subgenus Profundulus and Tlaloc (Teleostei: Profundulidae). Int. J. Morphol., 32(3):1074-1078, 2014.

SUMMARY: The genus Profundulus is one of the oldest fish groups, distributed north of Central America in Mexico, Guatemala and Honduras. However, there are few studies that determine the number of species and their phylogenetic relationships. Historically it has been recognized that this taxon comprises two natural groups, defined as the subgenus Profundulus and Tlaloc. Recent genetic researches have revealed a high degree of differentiation between the two taxa and some species, probably associated with the morphological changes. In this way, we made the osteological comparison between six species of the subgenus Profundulus and Tlaloc (Cyprinodontidae: Profundulidae). We reviewed 17 bones located in the skull and pelvic girdle; qualitative differences were found in eight bones: the mesethmoid, vomer, lachrymal, premaxilla, articular, quadrate, interoperculum, and basipterygium. The variation patterns permit to diagnose both subgenus, corroborating the previous morphological and molecular studies. The evidence suggests that both taxa could be relocated as genus, such as previously was established.

KEY WORDS: Cyprinodontiformes; Middle America; Freshwater fishes; Comparative osteology; Neurocranium; Branchiocranium.

\section{REFERENCIAS BIBLIOGRÁFICAS}

Álvarez, J. \& Carranza, J. Descripción de un género y especie nuevos de peces ciprinodóntidos procedentes de Chiapas (México). Cienc., 11:40-2, 1951.

Costa, W. J. E. M. Phylogeny and Classification of the Cyprinodontiformes (Euteleostei: Atherinomorpha): A Reappraisal. In: Malabarba, L. R.; Reis, R. E.; Vari, R. P.; Lucena, Z. M. S. \& Lucena, C. A. S. (Eds.). Phylogeny and classification of neotropical fishes. Porto Alegre, Edipucrs, 1998. pp.537-60.

Doadrio, I.; Carmona, J. A.; Martinez, E. \& De Sostoa, A. Genetic variation and taxonomic analysis of the subgenus Profundulus. J. Fish Biol., 55(4):751-66, 1999.

Espinosa, P. H.; Gaspar, D. M. T. \& Fuentes, M. P. Listados faunísticos de México. III. Los peces dulceacuícolas mexicanos. México D. F., Instituto de Biología, U.N.A.M., 1993.

González-Díaz, A. A.; Díaz-Pardo, E.; Soria-Barreto, M. \& RodilesHernández, R. Análisis morfométrico de los peces del grupo labialis, género Profundulus (Cyprinodontiformes:
Profundulidae) en Chiapas, México. Rev. Mex. Biodivers., 76(12):55-61, 2005.

Hollister, G. Clearing and dyeing fish for bone study. Zool., 12(10):89102, 1934.

Hubbs, C. L. Studies of the fishes of the Order Cyprinodontes. Misc. Publ. Mus. Zool., Univ. Mich., (13):1-31, 1924.

Martínez, E. Taxonomía y Zoogeografía de la Ictiofauna Dulceacuícola del Estado de Oaxaca, México (Microforma). Tesis doctoral microfichas $N^{\circ} 3700$. Barcelona, Universidad de Barcelona, 2000.

Matamoros, W. A. \& Schaefer, J. F. A new species of Profundulus (Cyprinodontiformes: Profundulidae) from the Honduran central highlands. J. Fish Biol., 76(6):1498-507, 2010.

Matamoros, W. A.; Schaefer, J. F.; Hernández, C. L. \& Chakrabarty, P. Profundulus kreiseri, a new species of Profundulidae (Teleostei, Cyprinodontiformes) from northwestern Honduras. Zookeys, (227):49-62, 2012.

Miller, R. R. A systematic review of the Middle American fishes of the genus Profundulus. Misc. Publ. Mus. Zool., Univ. Mich., (92):1-64, 1955.

Miller, R. R.; Minckley W. L. \& Norris S. M. Freshwater fishes of México. Chicago, Chicago University Press, 2005.

Nelson, J. S. Fishes of the World. New York, John Wiley \& Sons, 2006.

Page, L. M. \& Burr B. M. A Field Guide to Freshwater Fishes of North America North of Mexico. Boston, The Peterson Field Guides Series - Houghton Mifflin Co., 1991.

Parenti, L. R. A phylogenetic and biogeographic analysis of Cyprinodontiform fishes (Teleostei, Atherinomorpha). Bull. Am. Mus. Nat. Hist., 168:335-557, 1981.

Uyeno, T. \& Miller, R. R. Relationships of Empertrichthys erdisi, a Pliocene Cyprinodontid fish from California, with remarks on the Fundulinae and Cyprinodontinae. Copeia, 1962:520-32, 1962.

Dirección para Correspondencia:

Alfonso Ángel González Díaz

El Colegio de la Frontera Sur

Unidad San Cristóbal

Carretera Panamericana y Periférico sur $\mathrm{s} / \mathrm{n}$

San Cristóbal de Las Casas, c.p. 29290

Chiapas

MÉXICO

Email: aangelgd@gmail.com

Recibido : 08-01-2014

Aceptado: 12-07-2014 